\title{
The Effects of Interval Circular Training on Blood Pressure and Lipid Profile in People with Hypertension
}

\author{
1Mahdiyeh Shamizadeh \\ 2Mahdi Jafarlu \\ ${ }^{3}$ Mohammad reza Zolfaghari \\ ${ }^{1}$ Master of sport Physiology, Urmia University, Iran \\ ${ }^{2} P h D$ student of Sport Physiology, Urmia University, Iran \\ ${ }^{3}$ Assistant Professor of Exercise Physiology, Faculty of Physical Education and Sport Sciences, Urmia University, Iran
}

\section{Doi:10.5901/mjss.2016.v7n4s2p69}

\begin{abstract}
Physical activity is an effective way to prevent and treat cardiovascular risk factors, especially high blood pressure and high blood fat. The primary aim of this study was to investigate the Advantages and Disadvantages of interval circular training on blood pressure and lipid profile in adults with primary blood pressure. 30 adults, including Men and Women with primary blood pressure (ages 42.28 \pm 4.42 ) were selected and randomly assigned to one of the two groups: 12 participant in control and 18 participant in training group. The training group exercised interval circular training 3 times per week for 8 weeks. Blood samples were taken before the experimentation and after two months from all of the individuals. Anthropometric and systolic blood pressure (SBP), diastolic (DBP), mean arterial blood pressure (MAP) and serum lipids were assessed. The gathered data were analyzed using paired and independent $t$-test with significant level $P<0.05$ using SPSS software. In training group compared to before the experiment, systolic, diastolic and mean arterial blood pressure was significantly decreased and significantly increased in HDL-C and total cholesterol and LDL-C and Triglycerid ratios (LDL / HDL) and (Tchol / HDL) were significantly decreased. In training group compared to non-training people, non-substantial decreases in blood pressure $(P<0.05)$ and substantial changes in lipid profile was observed $(P<0.05)$. Our findings indicates that interval circular training can lower and improve blood pressure and lipid profile in middle-aged adults with hypertension.
\end{abstract}

Keywords: Combined training, High blood pressure, high density lipoprotein

\section{Introduction}

Nowadays the pattern of diseases has changed from infectious diseases to non-communicable such as cardiovascular, diabetes, and cancer (Mestek, Garner, Plaisance, Taylor \& Grandjean, 2006). Cardiovascular disease is the cause of early death worldwide in both the men and women (Joep, Guy \& et al, 2012), and these diseases have a lot of costs directly and indirectly (Murray, Lauer, \& et al, 2003). Cardiovascular risk factors that lead to death are placed on high blood pressure, tobacco use, high blood glucose, physical inactivity, high cholesterol, overweight and obesity (Centers for Disease \& Prevention, 2001). High blood pressure is the most common cause of cardiovascular diseases (CVD) in the world (Gomes, 2012). Every year, nearly 1.7 million deaths worldwide are because of high blood pressure. It is predicted that in 2025, there will be about 1.56 billion adults who suffer from high blood pressure (World Health Organization, 2012).

Hyperlipidemia, or high cholesterol, is another risk factor for cardiovascular diseases (Holewijn, Heijer, Stalenhoef, 2010). It is estimated that high cholesterol causes 6.2 million deaths a year and it is the reason of about 2 percent of the total number of daily deaths in the world (Centers for Disease \& Prevention, 2001). The extensive literature on the effects of fats in atherosclerosis is available. The importance of lowering the cholesterol is mentioned in several studies in primary and secondary prevention strategies to reduce cardiovascular risk factors (Nadar, Lim, Beevers, Lip, 2002 \& Wierzbicki, 2007).

There are strong evidences that in many developing countries such as Iran, the prevalence of hypertension (HTN), obesity and followed by that, cholesterol and heart disease is increasing (Haghdoost, Sadeghirad, Rezazadeh, 2008). This rapid increase in Iran and other developing countries is related to the low or lack of physical activity (Sarrafzadegan, Rabiei, Kabir, Asgary \& et al, 2007). Wilson and his colleagues showed that aerobic exercises for 10 weeks is effective 
on patients with hypertension to decrease their blood pressure, while aerobic exercise with $60 \%$ intensity is not better than an aerobic training program with $20 \%$ intensity (1999). Nelson and his colleagues (2013) reported that the combination of endurance and resistance training have significantly more positive effects on changes in blood pressure in older patients with primary hypertension. William and partners mentioned that the LDL-C is more influenced with aerobic exercise and more significant effect to above $70 \%$ of maximum heart rate intensity to the cholesterol concentration (2002). Paul reported that the two months of combined training of endurance and resistance did not have any significant changes in the ratio of TC/HDL-C results, while Julia and et al reported the significant changes in contradiction with the previous results (Paul, 2004).

Referring to the preceding studies, it is obvious that there is not enough research on the effects of circular interval training as a new method of regulating the blood pressure. Therefore, this study was designed to research the effects of combined training and interval exercises on blood pressure and blood lipid profile in middle-aged people with primary hypertension.

\section{Research Methods}

This is a semi-experimental research and society is made up of men and women 35-55 years old with primary hypertension. We chose 30 targeted cases from 39 of the patients of tabriz health centers to participate in this training program. Inclusion criteria were: systolic blood pressure 140-159 mmHg and diastolic blood pressure 90-99 $\mathrm{mmHg}$ and the exclusion criteria for the study included chronic diseases and smoking and uncontrolled hypertension. Subjects didn't have regular physical activity in the past year and least one year had been passed of diagnosis by the doctor and they took no medication to control blood pressure or only taking medications for cross-sectional. During the study, every patient was controlled once every two weeks to monitor blood pressure were under the supervision of a physician. During the meeting of familiarization with the practice environment and measurement of Anthropometric properties, people were randomly grouped in to the control group $(n=12)$ and exercise $(n=18)$ and their Systolic and diastolic and mean arterial blood pressure was measured.

\subsection{Training protocol}

The training program consisted of interval training on the ergometer bicycle followed by resistance training in four stations. Each session this circular protocol was repeated for three times. Training Group's program was for 8 weeks, 3 sessions per week and each session was 75 minutes, while the control group did not follow the training program. Every training session included warm-up involving 10 min low-intensity walking and and 5 min cool-down light stretching at the end of training.

The participants trained by alternating $8^{\prime}$ of aerobic exercise on cycloergometer (for $3^{\prime}$ at $50 \%$ and $1^{\prime}$ at $75 \% \mathrm{HRR}$ (50-70 rpm)), with training at four resistance exercise stations after 1 min recovery. Subjects wait 30" between one station and the following. After concluded one complete round subjects repeat all another two times with 3 ' rest between rounds.

Intensity of training on the bike was calculated on the basis of heart rate reserve using the Karvonen formula using a Polar heart rate monitor (Friedwald, Levy, Fredrickson, 1972). Stations intended for resistance training included: underhand cable pulldowns, hip extension, lateral shoulder raise and leg press. Training in stations was at the intensity of $70 \% 6 \mathrm{RM}$ with the rest pause method as: six reps, 20 " recovery, two reps, 20 " recovery then one or two more reps until exhaustion has been reached.

Heart rate reserve $(\mathrm{HRR})=$ Maximum heart rate- Resting heart rate (HRrest) $(1)$

Maximum heart rate $(\mathrm{MHR})=220$-Age $(2)$

\subsection{Measuring blood samples}

Before and after the training period, blood samples were taken at the morning and after an overnight fast from all subjects. Samples were kept at $-80^{\circ} \mathrm{C}$ for subsequent analysis. Tritriglyceride (TG), total cholesterol (Tchol), high-density lipoprotein cholesterol (HDL-C) and LDL-C were measured (mg/dl) by a photometric method using Japan's 717 Hitachi analyzer.

LDL-c fraction was calculated from Friedewald's formula: $L D L C=T C-H D L C-(T G / 5)$ 


\subsection{Statistical analysis of data}

In this study, we used descriptive statistics to describe the variables and inferential statistics were used to analyze the data. Data results were expressed as a mean \pm standard deviation. The Shapiro-Wilk test was used to determine the normal distribution of data. Anything was analyzed using SPSS 20 at a significance level of five percent ( $P \leq 0.05)$.

\section{Analysis and Results}

Physiological characteristics of participants has been shown in Table 1.

Table 1. Subjects Characteristics

\begin{tabular}{lcc}
\hline & Control & Training \\
\hline Age(year) & $41.5 \pm 4.08$ & $43.06 \pm 4.76$ \\
Height(cm) & $170.42 \pm 8.8$ & $171 \pm 8.24$ \\
Weight $(\mathbf{k g})$ & $79.25 \pm 6.85$ & $82.08 \pm 12.02$ \\
BMI(kg/m $)$ & $27.5 \pm 3.8$ & $28.05 \pm 3.44$ \\
Body fat mass(kg) & $20.96 \pm 2.56$ & $21.75 \pm 3.84$ \\
\hline
\end{tabular}

Results related to hemodynamic parameters (Table 2) showed that interval Circular training is significantly reduced resting heart rate compared with control group $(P=0.05)$. Comparisons between the two groups, there were significant changes in systolic blood pressure $(P=0.148)$, diastolic blood pressure $(P=0.077)$ and mean arterial blood pressure $(P=0.06)$ (Table 2). Results obtained within group t-test showed that interval circular training significantly reduced systolic blood pressure $(P<0.001)$, diastolic blood pressure $(P<0.001)$ and mean arterial blood pressure $(P<0.001)$ but no significant changes were observed in the control group.

Table 2. Average descriptive characteristics and blood parameters before and after in two groups

\begin{tabular}{lccccc}
\hline & Groups & Pre & Post & $\begin{array}{c}\text { P-value } \\
\text { Within group }\end{array}$ & $\begin{array}{c}\text { P-value } \\
\text { Between group }\end{array}$ \\
\hline Weight $(\mathbf{k g})$ & Control & $79.25 \pm 6.85$ & $79.46 \pm 6.59$ & 0.333 & 0.791 \\
& Training & $82.08 \pm 12$ & $78.46 \pm 11.7$ & $*<0.001$ & \\
Body fat mass $\mathbf{( k g})$ & Control & $20.96 \pm 2.56$ & $20.98 \pm 2.45$ & 0.868 & 0.635 \\
& Training & $21.75 \pm 3.84$ & $20.4 \pm 3.65$ & $*<0.001$ & \\
HR $_{\text {rest }}(\mathbf{p b m} / \mathbf{m i n})$ & Control & $76.33 \pm 5.53$ & $76.25 \pm 5.86$ & 0.82 & $* 0.05$ \\
& Training & $75.5 \pm 6.18$ & $71.89 \pm 5.64$ & $*<0.001$ & \\
$\mathbf{S B P}(\mathbf{m m H g})$ & Control & $145.92 \pm 4.7$ & $145.33 \pm 5.1$ & 0.501 & 0.148 \\
& Training & $148.94 \pm 14.39$ & $140.17 \pm 13.25$ & $*<0.001$ & \\
$\mathbf{D B P}(\mathbf{m m H g})$ & Control & $92.75 \pm 2.05$ & $92.25 \pm 2.26$ & 0.377 & 0.077 \\
& Training & $95.56 \pm 5.56$ & $89.89 \pm 4.04$ & $*<0.001$ & \\
$\mathbf{M A P}(\mathbf{m m H g})$ & Control & $110.47 \pm 1.75$ & $109.94 \pm 2.22$ & 0.355 & 0.06 \\
& Training & $112.07 \pm 6.55$ & $106.65 \pm 6.52$ & $*<0.001$ & \\
\hline
\end{tabular}

* indicates significance changes

Independent t-test between the control and exercises group showed a significant reduction in the Triglycerides $(\mathrm{P}=0.018)$, total cholesterol $(P=0.001)$, LDL-C $(P=0.042)$, Tchol/HDL $(P<0.001)$, LDL/HDL $(P<0.001)$ and a significant increase in levels of $\mathrm{HDL}(\mathrm{P}<0.001)$ showed (table 3$)$. In addition to in the exercise group compared to before activity, significant changes were observed in profile blood fat, but the level of triglycerides, total cholesterol, LDL-C, HDL-C, Tchol / HDL and LDL / HDL for the control group was not significant compared to the prior. 
Table 3. Average changes in lipid profile in both groups before and after exercise

\begin{tabular}{lccccc}
\hline & Groups & Pre & Post & $\begin{array}{c}\text { P-value } \\
\text { Within group }\end{array}$ & $\begin{array}{c}\text { P-value } \\
\text { Between group }\end{array}$ \\
\hline TG(mg/dl) & Control & $188 \pm 35.95$ & $191.75 \pm 51.12$ & 0.63 & $* 0.018$ \\
& Training & $192.39 \pm 77.29$ & $144.56 \pm 4965$ & $*<0.001$ & \\
Tchol(mg/dl) & Control & $206.83 \pm 12.67$ & $209.83 \pm 17.01$ & 0.292 & $* 0.001$ \\
& Training & $204.5 \pm 24.09$ & $179.67 \pm 24.79$ & $*<0.001$ & \\
HDL(mg/dl) & Control & $40 \pm 1.6$ & $40.08 \pm 1.56$ & 0.809 & $*<0.001$ \\
& Training & $41.22 \pm 4.92$ & $47.72 \pm 5.46$ & $*<0.001$ & \\
LDL(mg/dl) & Control & $129.67 \pm 13.84$ & $131.83 \pm 17.21$ & 0.436 & $* 0.042$ \\
& Training & $129.63 \pm 37.49$ & $109.06 \pm 34.09$ & $*<0.001$ & \\
LDL/HDL & Control & $3.26 \pm 0.28$ & $3.39 \pm 0.5$ & 0.277 & $*<0.001$ \\
& Training & $3.2 \pm 0.86$ & $2.21 \pm 0.69$ & $*<0.001$ & \\
Tchol/HDL & Control & $5.18 \pm 0.28$ & $5.29 \pm 0.6$ & 0.329 & $*<0.001$ \\
& Training & $5.13 \pm 0.91$ & $3.97 \pm 0.75$ & $*<0.001$ & \\
\hline
\end{tabular}

* indicates significance changes

Systolic and diastolic blood pressure and mean arterial blood pressure after the training period in exercise group compared to the control group was statistically significantly decreased but the level difference of Average systolic blood pressure and diastolic and mean arterial blood pressure before and after exercise Between two groups was not significant (Figure 1).

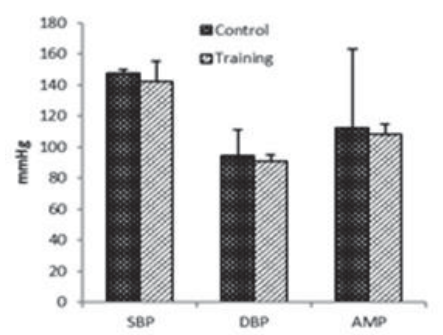

Figure 1. Compares the mean difference of blood pressure between the two groups after 8 weeks of training

In both groups, differences in levels of triglyceride after exercise compared to baseline was significant. In values of LDL and ratios of Tchol/HDL and LDL/HDL significantly reduction between exercise and control groups was observed (Figure 2 and 3). The concentration of HDL compared between the two groups significantly increased (Figure 2).

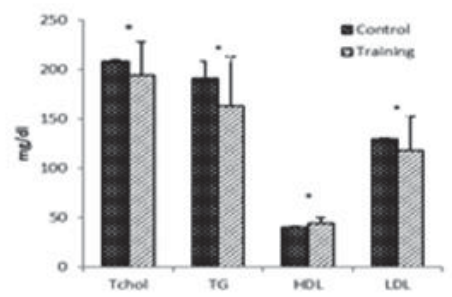

Figure 2. Compare the mean concentrations differences of blood lipid profile between the two groups after 8 weeks of training 


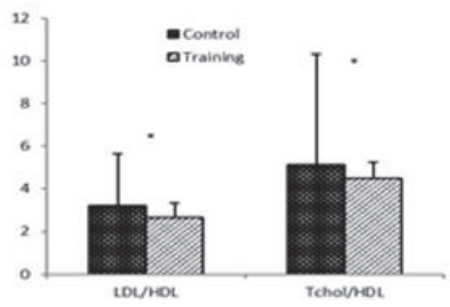

Figure 3. Compare the mean differences of blood lipid ratios between the two groups after 8 weeks of training

\section{Discussion}

In this study, men and women aged 30-50 years assigned two groups, exercise (with interval circular training) and control group (no exercise). Blood samples for the study of lipid profile and blood pressure were obtained in two stages (before and after 8 weeks of training). The main finding of this study was the effect of interval circular training on blood pressure reduction and blood lipids improvement in midlle-aged individuals with hypertension.

The results of this study showed that eight weeks of training in the form of the combination of interval and circular training, significantly reduced systolic and diastolic and mean arterial hypertension compared to before exercise. This finding are consistent with the results of investigations Carmen (1990), Stone (1991), Wilson (1999), Vroniko (2004), Rousseau (2011), Markiov, (2013), Nelson (2013), Naghi-ei (1390), Land (1391) and his colleagues. The long-term Combined exercises with Moderate and higher intensity due to increases capillary network in the active muscles and increases the flexibility of the vessels and by lowering the stimulation of the sympathetic nerve and reduce artery retractor hormone and the above all Constrictor peptide endothelin-1 that is a vascular endothelial cells and may lead to Increased production of nitric oxide (NO), so, it can be a perfect solution to reduce the systolic and diastolic blood pressure (Gregoski, Barnes, Tingen, Harshfield, Treiber, 2011). It seems intermittent exercise has a better effect on arterial stiffness and blood pressure control in people with high blood pressure. since that part of the subjects' training were conducted as alternate on ergometer Bicycle, there is also a possibility of lowering blood pressure through this mechanism. The mechanisms involved in arterial stiffness are elastic matrix degradation, endothelial dysfunction, hypertrophy and Smooth muscle cell hyperplasia and increase the amount of collagen (Guilherme, Emmanuel \& et al, 2010). Changes in blood lipid profile is Another mechanism that Physical exercises with the impact on them can lead to improve blood pressure that was an important finding of this study. Exercise leads to the activation of lipase cellular enzymes And by reducing LDL-C, chylomicrons, triglycerides and total plasma cholesterol and increase levels of concentration HDL-C can have anti-hypertensive effects (Arimoto, Kijima, Muramatsu, 2005).

In contrast with results of this research can be noted the investigations conducted by Hagbrg (1989), Regress (1996), Hunter and McCarthy (2000), Frestine (2006), Mortimer (2011) and Amanda (2013). The Contradiction in results can be related to the type of exercise, intensity and duration of activity.

In relation to lipid profile, results showed that the 8 weeks of combined training as circular and alternate with relatively high intensity, can cause a significant reduction in triglycerides (TG), total cholesterol (Tchol), low-density lipoprotein (LDL) and ratios (LDL/ HDL) and (Tchol / HDL) and also a significant increase in high density lipoprotein (HDL) in active people with hypertension than before the activity. As well as significant improvement was observed in blood lipid profile with performing 8 weeks training compared with 8 weeks of non-training (Ming-Lang \& et al 2013., Nash, 2001., Robert, 2006., Casella, 2001., Antonio, 2013 ). Consistent with this study findings, Antonio and Robert have shown that 4-month training program in over weighted men with high blood pressure improves the Functional characteristics of HDLC (Roberts, Hama, Eliseo, 2006 \& Casella, Chagas \& et al, 2011). Fahlman (2002) showed that exercise increased levels of concentration (HDL-C) in women (Nash, Jacobs, 2001). The results of the studies of Arazi and his colleagues (2013) showed that 8 weeks of combined training had a significant reduction in total cholesterol, triglycerides and low-density cholesterol (LDL) and a significant increase in high-density cholesterol (HDL) in middle-aged men.

The results of this study contradict the results of studies that carried out by Cole (1992), Smotok (1993), Linda (2000), Brusha (2000), Velsman (2000), Key (2003), Tambalys (2009) and Magni (2010). Smotok and colleagues showed that there is no significant changes in blood lipid profile after strength training (Ming-Lang, CHien, SHih-chang, 2013). Velsman and et al, showed that after 8 weeks of training on a treadmill at 80 percent of maximum heart rate, no changes 
observed in total cholesterol and HDL. The reason for the changes in triglycerides (TG) in serum is increase the activity of lipoprotein lipase (LPL). It seems that combined training despite resistance training increased activity of this enzyme. LPL causes the release of fatty acids from adipose tissue and muscles and catabolism of TG and triglyceride-rich lipoprotein increases and facilitates the removal of TG from the blood flow. Even no change in body composition decreased TG and this confirms this mechanism (Yektayar, Mohammadi, Ahmadi, Khodamoradpour, 2012). During the exercise, and after that, the insulin plasma is reduced and probably it is one of the factors that changes the cholesterol. A reduction on insulin, activates lipolysis of adipose tissue and increases the concentration of free fatty acid in plasma. Along with the decrease in insulin, glucagon secretion increases that accelerate the process of lipolysis (Arazi, Jorbonian, Asghari, 2013).

It is known that the endogenous synthesis of testosterone, is one of the main factors of increasing HDL levels a result of resistance training (Arazi, Jorbonian, Asghari, 2013). According to the results of this study, rise of HDL levels occurred after combined training. Various factors affect changes in blood HDL. It seems that weight, sex, protocol and duration of the exercise, is a key factor in response of HDL to combined training (Yektayar \& et al, 2012). Physiologically, the reason for the increasing of HDL can be attributed to factors such as increase of cholesterol Lecithin acyltransferase enzyme(LCAT), Lipoprotein lipase (LPL) and reduction activity of hepatic lipase (HTGL). It is reported that the liver through its nuclear receptors such as LXR that are known as intracellular sensors in response to Too much cholesterol in HDL stimulate reverse cholesterol transport process and transport it to the liver, The convesion of cholesterol to bile acids and disposed it. Increase the expression gene of LXR due to aerobic combined training is one of the mechanisms that enhance HDL activity (Tartibian, Yaghoobnezhad, Saboory, Kheradmand, 2013).

\section{Conclusions}

Overall, it was concluded that circular physical activity with relatively high intensity have positive effects in people with primary hypertension and reducing their blood pressure and improving blood lipid profile and Reducing risk factors for cardiovascular disease in middle age and later in life. However, lack of full-time control on these people's diet and sleep were limitations of this study that causes caution in generalizing the results of this study. Also further investigations requires to study the effective mechanisms of physical activity with different intensity and duration and different volumes on blood pressure and blood lipid profile.

\section{References}

Amanda, M.R., Gregory, M., \& et al.(2013). The Impact of Resistance Training on Resting Blood Pressure in Adults as an Example, Canadian Journal of Cardiology, 29 ,622-627.

Anaerobic threshold, is it a magic number to determine fitness for surgery?. (2013) Older Perioperative Medicine, 2:2.

Antonio, P., Pacelli,,T.M., Giuseppe, M., Marco, N., Giuseppe, B.,Gi, Francesco, B., \& Antonino, B.(2013). Effects of high-intensitycircuit training,low-intensity circuit training and endurance training on blood pressure andlipoproteins in middle-aged overweight men , Lipids in Health and Disease,12,131.

Arazi, H., Jorbonian, A., Asghari, E.(2013). Comparison of Concurrent (Resistance-Aerobic) and Aerobic Training on VO2max Lipid Profile, Blood Glucose and Blood Pressure in Middle-Aged Men at Risk for Cardiovascular Disease, JSSU,20 (5) :627-638.

Arimoto, M., Kijima, A., Muramatsu, SH.(2005). Cardio respiratory responses to dynamic and static leg press exercise in humans, $J$ Physiol Anthropol Appl Hum Sci, 24(4), 277-83.

Carmen, C.C., ,Michael, L.P., \& et al.(1991). Effect of exercise training on blood pressure in 70-to 79-yr-old men and women , Medicine and science in sports and exercise.

Casella-Filho, A., Chagas, AC., Maranhão, RC., Trombetta, IC., Cesena, FH., Silva, VM., Tanus-Santos, JE., Negrão, CE., da Luz, PL. (2011), Effect of Exercise

Training on Plasma Levels and Functional Properties ofHigh-Density Lipoprotein Cholesterol in the Metabolic Syndrome, Am $J$ Cardiol,107(8),1168-1172.

Centers for Disease Control and Prevention.(2001). National Center for Chronic Disease Prevention and Health Promotion. Office on Smoking and Health.

Friedwald, WT., Levy, RI., Fredrickson ,DS. (1972). Estimation of the concentration of low density lipoprotein in plasma without use of preparative ultra centrifug, Clin chem,18.499-502.

Gaesser, G.A., and Rich, R.G .(1984). Effects of high-ad low intensity exercise training on aerobic capacity and blood lipids, Med. Sci. Sports Exercise, 16,269-574.

Gomes, C.E. (2012). High-intensity interval training and hypertension: maximizing the benefits of exercise? . Am J Cardiovasc Dis, 2(2), 102-110.

Gregoski, M.J., Barnes, V.A., Tingen, M.S., Harshfield, G.A., Treiber, F.A.(2011). Breathing awareness meditation and LifeSkills 
Training programs influence upon ambulatory blood pressure and sodium excretion among African American adolescents, $J$ adolesc health, 48(1), 59-64.

Guilherme, V.G., Emmanuel, G.C., \& et al.(2010). Effects of continuous vs. interval exercise training on blood p ressure and arterial stiffness in treated hypertension, Hypertension Research, 33, 627-632.

Haghdoost, A., Sadeghirad, B., Rezazadehkermani.,M.(2008). Epidemiologyand Heterogeneity of Hypertension in Iran , A Systematic Review.Arch Iranian Med, 11 (4), $444-452$.

Heidi, K., Byrnel \& Jack, H.W.(2000). The Effects of Resistance Training on Resting Blood Pressure in Women, Journal of Strength and Conditioning Research, 14(4), 411-418.

Holewijn,S., Heijer, S., \& Stalenhoef, A. H. (2010). Apolipoprotein B, non-HDL cholesterol and LDL cholesterol for identifying individuals at increased cardiovascular risk, Journal of Internal Medicine, 268, 567-577.

Joep, P., Guy D \& et al. (2012). European Guidelines on cardiovascular disease prevention in clinical practice. European Heart Journal, $33,1635-1701$.

John, D., Eicher, B., Carl, M., Maresh, G., Tsongalis, P., Thompson, MD., Linda, S. (2010) The additive blood pressure lowering effects of exercise intensity on post- exercise hypotension, AmHeart,160,513-20

Julia, KW., Roberts, CK., Tung, H., Barnard RJ.(2004). Effect of diet and exercise intervention on inflamnatory and adhesion molecules in postmenopausal women on hormone replacement therapy and at risk for coronary artery disease, Metabolism,53,377-381.

Magni, M., Nikolai, B.N., \& et al.(2014). High-Intensity Intermittent Swimming Improves Cardiovascular Health Status f or Women with Mild Hypertension, BioMed Research International.

Mestek, M., Garner, G.C., Plaisance, E.P., K.Taylor, J., Alhassan, S., \& W. Grandjean, P. (2006). Blood lipid responses after continuous and accumulated aerobic exercise . Journal of Sport Nutrition and Exercise Metabolism ,16, 245-54.

Ming-Lang, T., Chien-Chang, Ho., Shih-Chang, C.(2013). A Simple Method for Increasing Levels of High-Density Lipoprotein Cholesterol, International Journal of Sport Nutrition and Exercise Metabolism, 23, 271 -281.

Mota, M.R.,Oliveira, D., Ricardo, J., \& et al.(2013). Acute and Chronic Effects of Resistive Exercise on Blood Pressure in Hypertensive Elderly Women, Journal of Strength \& Conditioning Research, 27(12) 3475-3480.

Murray, CJ., Lauer, JA., Hutubessy, RC., Niessen, L., Tomijima, N., Rodgers, A., Lawes CM., et al. (2003). Effectiveness and costs of interventions to lower systolic blood pressure and cholesterol: a global and regional analysis on reduction of cardiovasculardisease risk. Lancet, 361,717-25.

Nadar, S., Lim, H.S., Beevers, D.G., Lip GY.(2002). Lipid lowering in hypertension and heart protection: observations from the Anglo Scandinavian Cardiac Outcomes Trial (AS C OT ) and the Heart Protection S tudy, J Hum Hyper tens, 16,815-817.

Naghii , M.R., Almadadi, M.(2011). Effect of Regular Physical Activity as a Basic Component of Lifestyle Modification on Reducing Major Cardiovascular Risk Factors, Knowledge \& Health , 6(1),27-35

Nash, MS., Jacobs, PL. (2001). Circuit resistance training improves the atherogenic lipid profiles persons with chronic paraplegin, $J$ Spinal Cord Med,24,2-9.

Nelson, S., Romeu, M., Catarina, A., Jaime, S., José, O.(2013). A randomized 9-month study of blood pressure and body fat responses to aerobic training versus combined aerobic and resistance training in older men, Experimental Gerontology.

Paul, DT. (2004). Apolipoprotein egeno type and maximal oxygen uptake with exercise training, Metabolism,53,193-202.

Recommendation of the second joint task force of Europen and other societies on coronary prevention. (1998). Eur Heart J,19,1434-503.

Robert, H., Fagard, D.(2000), Exercise characteristics and the blood pressure response to dynamic physical training, American College of Sports Medicine.

Roberts, CK., Hama, S., Eliseo, AJ., Barnard, RJ.(2006). Effect of ashort-term diet and exercise interventionon inflammatory/antiinflammatoryproperties of HDL in overweight/obese men with cardiovascularrisk factors , J Appl Physiol,101,1727-1732.

Salvi, P.(2012). How vascular Hemodynamics affects blood pressure, springer,978-88-470.

Sarraf zadegan, N. , Rabiei, K., Kabir, A., Asgary, S. ,and et al.(2007). Changes in lipid profile of patients referred to a cardiac rehabilitation program, European Journ al of Cardiovascular Prevention and Rehabilitation, 15, , 4 67-472.

Stone, M.H., Fleck, SJ., Triplett, NT., Kraemer, WJ.(1991). Health- and performance-related potential of resistance training, Sports Med, 11(4), 210-31.

Tartibian B, Yaghoobnezhad F, Saboory E, kheradmand F.(2013) The Response of serum cortisol and lipid profile to a moderately intensive aerobic exercise in non- active middle age men, journal of urmia university of medical sciences,24 (6) :393-404

Wallace, M.B., Moffatt, R.J., Haymes, E.M \& et al.(1991). Acute effects of resistance exercise on parameters of lipoprotein metabolism, Medicine and Science in Sports and Exercise, 23, 199-204.

Wierzbicki, A.S. (2007). Lipid-altering therapies and the progression of atherosclerotic disease, Cardiovasc Intervent Radiol, 30,155-16 0.

William, E., Kraus, M.d.,Joseh, A., Houmard,Braind., et.al. (2002). Effect of omount and intensity of exercise on plasma lipoproteins, The New England Journal of Medicine,347,1483-1492.

Wilson, D., Moreira,Flávio, D., Fuchs, P., \& Lawrence, J.A. (1999). The Effects of Two Aerobic Training Intensities on Ambulatory Blood Pressure in Hypertensive Patients:Results of a Randomized Trial , J ClinEpidemiol , 52, 637-642.

World Health Organization. (2012). Regional Office for Southeast Asia, Hypertension fact sheet. Hypertension.

Yektayar, M., Mohammadi, S., Ahmadi Deharshid, K., Khodamoradpour, M.(2012). Comparison of the effects of resistance, endurance and combined exercise on lipid profile of non- athlete healthy middle aged men, journal of Kurdistan university of medical sciences, 16(4), 26-36. 